\title{
UN SISTEMA DE WOOC PARA LA ACTUALIZACIÓN DOCENTE
}

A WOOC SYSTEM FOR TEACHING UPDATE

\section{CARLOS BRAVO REYES}

Universidad Autónoma Gabriel René Moreno

BOLIVIA

El juego de la verdad y del error no sólo se juega en la verificación empírica y la coherencia lógica de las teorías; también se juega a fondo en la zona invisible de los paradigmas. Esto lo debe tener bien en cuenta la educación. Edgar Morin 


\begin{tabular}{cc}
\hline \multicolumn{1}{c}{ CARLOS BRAVO REYES } \\
\\
$\begin{array}{cc}\text { UN SISTEMA DE WOOC PARA LA ACTUALIZACIÓN DOCENTE } \\
\text { Págs. } 75 \text { - 87. DOI: } h \text { http://dx.doi.org/10.30972/rfce.0203255 }\end{array}$ \\
\hline & Recibido: $03 / 09 / 2018$ \\
& Aceptado: $12 / 10 / 2018$
\end{tabular}

\title{
RESUMEN
}

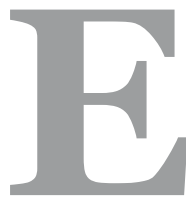

1 artículo valora la utilidad de emplear WhatsApp para la superación de los profesores universitarios, a partir del desarrollo de un sistema de Wooc (WhatsApp Open On line Course). La base de este sistema de superación es el mobil learning, el que se desarrolla a partir de micro contenidos soportados en micro medios. Se realiza una discusión conceptual de los términos anteriores. En el caso del sistema de cursos partimos de un diseño instruccional propio, donde el micro contenido y los micro medios se conciben de manera diferente, estos últimos tienen funciones diversas como es el caso de la informativa, problémica, de retroalimentación y conclusiva. Los micro medios se elaboran combinando imágenes y texto, para que la información sea precisa, leída sin necesidad de agrandar la imagen en el celular y de fácil comprensión. El sistema de Wooc compuesto por doce cursos, fue evaluado como satisfactorio por la mayoría de los participantes.

Palabras clave: micro aprendizaje, aprendizaje móvil, Wooc, micro contenidos, micro medios.

\begin{abstract}
The article assesses the usefulness of using WhatsApp for the improvement of university professors, based on the development of a Wooc system (WhatsApp Open On line Course). The basis of this system of improvement is the mobile learning, which is developed from micro content supported in micro media. A conceptual discussion of the previous terms is carried out. In the case of the system of courses, we start with our own instructional design, where the micro content and the micro media are conceived differently, the latter have different functions: informative, problem, feedback and conclusive. The micro media are made by combining images and text, so that the information is accurate, read without the need to enlarge the image on the cell phone and easy to understand. The Wooc system composed of twelve courses was evaluated as satisfactory by the majority of participants.
\end{abstract}

Keywords: micro learning, mobile learning, Wooc, micro content, micro media.

\section{INTRODUCCIÓN}

La Facultad de Humanidades es una de las 13 facultades que pertenecen a la Universidad Autónoma Gabriel René Moreno, institución pública de nivel superior que radica en el depar- 
tamento de Santa Cruz en Bolivia. La Universidad cuenta con una matrícula que sobrepasa los 105 mil estudiantes. Nuestra Facultad acoge a más de 10 mil estudiantes, en sus seis carreras en las que trabajan unos 220 profesores. La mayoría de ellos tienen diplomados y maestrías en áreas relacionadas con la docencia, pero la superación es asistemática, no existe un plan de formación continua y las veces que se realizan actividades de esta índole carecen de sistematicidad, con temas variados y poca participación de los profesores.

Desde los últimos meses del 2016 y por un mandato de la Asociación de profesores de nuestra Facultad empezamos a realizar un sistema de cursos de actualización en el campo de la tecnología e innovación educativa. Este sistema se apoya en la concepción del micro aprendizaje y toma a WhatsApp para su desarrollo. Con ese fin se creó un grupo en esta red que cuenta con 110 participantes de ellos 75 son profesores de la Facultad y el resto invitados de otras universidades.

El sistema de cursos bajo esta modalidad llamado WOOC, (WhatsApp Open Online Course o Curso abierto en línea a través de WhatsApp). Hasta el presente para el grupo antes mencionado se ejecutaron 12 cursos, que se desarrollan el último martes de cada mes. El Wooc puede ser revisado tanto desde su dispositivo móvil, como desde la aplicación de escritorio de WhatsApp.

El objetivo del trabajo está dirigido a demostrar la utilidad del Wooc como forma de organizar la actualización de los profesores en temas puntuales. El artículo se inicia con una conceptualización general del e-learnng, el m-learning y el micro aprendizaje. Posteriormente mostramos el diseño instruccional que se sigue en la elaboración de los Wooc y concluye con la valoración de los participantes en el sistema de cursos antes mencionados.

\section{DEL E-LEARNING AL M-LEARNING PARA COMPRENDER EL MICRO APRENDIZAJE}

Antes de ingresar al análisis conceptual, es necesario recordar que los medios de enseñanza, (desde la imprenta hasta los celulares actuales) tienen un lugar privilegiado en el proceso de aprendizaje. No es posible concebir este complejo proceso sin la existencia de uno o de varios medios. Por ello desde el momento en que la digitalización de la información se convirtió en un proceso cotidiano, al que le siguió la creación de numerosos dispositivos digitales, estos se emplearon de diferentes maneras en el complejo campo del aprendizaje. Para explicar la inserción de estos medios se crean diferentes conceptos como e-learning, mobil learning y micro learning, que significan, aprendizaje electrónico (o mediado por objetos electrónicos), aprendizaje móvil (mediado por dispositivos móviles) y micro aprendizaje (aprendizaje por etapas o en períodos cortos de tiempo)

Tal vez parezca contradictorio que sea el medio o recurso el que favorezca la creación de los conceptos anteriores, pero no debemos olvidar que estos medios primero se emplean fuera 


\begin{tabular}{cc}
\hline CARLOS BRAVO REYES \\
$>78$ & $\begin{array}{c}\text { UN SISTEMA DE WOOC PARA LA ACTUALIZACIÓN DOCENTE } \\
\text { Págs. } 75-87 . \text { DOI: } h \text { ttp }: / / d x \cdot d o i . o r g / 10.30972 / r f c e .0203255\end{array}$ \\
\hline
\end{tabular}

de la escuela y después se introducen en los sistemas escolares. Los plazos entre su empleo masivo y la inserción en las instituciones escolares cada día se acortan.

El e-learning se puede entender como la forma de explicar el aprendizaje mediado por diferentes tecnologías de información. (Conde, 2014). Cabero (2006) reconoce al e-learning como una estrategia formativa que tiene la posibilidad de contribuir a resolver problemas como la separación del estudiante a las instituciones escolares, la contribución permanente al desarrollo de la formación de la sociedad, el ahorro de tiempo y recursos.

Una consulta a la literatura especializada muestra que e-learning se define de maneras diferentes, (Vidal Ledo, 2015) desde paradigma (S.Coop, 2009), proceso (Conde, 2014) hasta estrategia (Cabero, 2006). En general se percibe que es el empleo de las tecnologías de la información y la comunicación por medio de una variedad de diferentes dispositivos y redes relacionadas con Internet. Sus inicios se limitaron al empleo de equipos con soporte digital, pero con Internet crecieron sus posibilidades. En lo que coinciden todos los autores analizados es en la formación del individuo, en su aprendizaje.

El e-learning es una forma de organizar el aprendizaje, con una relación didáctica entre contenidos, métodos y estrategias y los medios y recursos, estos últimos responsables de generar la (e) del concepto. Como estos evolucionan con gran rapidez es de esperar que el e-learning también se modifique continuamente. Por ello al introducir dispositivos móviles, se da paso a una nueva forma denominada m-learning, donde el celular es el recurso más importante.

Fue el 3 de abril de 1973 cuando Martin Cooper realizó la primera llamada desde un celular, cuya batería duraba solamente 20 minutos. Desde ese momento a la fecha el desarrollo de estos dispositivos es permanente y con ello la mejora en uno de sus principales atributos la ubicuidad. Como muchos dispositivos tecnológicos al cabo de los años encontró un espacio en la enseñanza. A finales de los 90 en EUA se inicia el empleo de las agendas electrónicas en la enseñanza y es el punto de partida para el aprendizaje móvil (Guerrero, 2011).

La investigación relacionada con el Mobile learning tiene sus inicios en los primeros años de este siglo (Gil, 2014) En la revisión bibliográfica se destacan tres momentos diferentes: el tecnocentrismo, la evolución a partir del E-Learning y por último el contexto de aprendizaje y por ende la persona (Pachelr, Bachmair, \& Cook, 2010).

El aprendizaje móvil implica el uso de tecnología móvil, sola o acompañada con otros tipos de tecnología, como las redes sociales. El aprendizaje se puede desarrollar en cualquier momento y lugar y con una variedad de formas (UNESCO, 2013). Se denomina aprendizaje móvil, en inglés, Mobile Learning, al proceso que vincula el uso de dispositivos móviles a las prácticas de enseñanza -aprendizaje en ambiente presencial o a distancia que permite, por un lado, la personalización del aprendizaje conforme a los perfiles del estudiante y por el otro, el acceso a contenidos y actividades educativas sin restricción de tiempo ni lugar. Con el aprendizaje móvil se aprovecha la convergencia digital de los dispositivos móviles en la capacidad de 
las aplicaciones que permiten registrar información de entornos reales; recuperar información disponible en la web y relacionar personas para realizar trabajo colaborativo (Aguilar, 2010).

El mobile learning define prácticas que emplean los dispositivos móviles y las tecnologías inalámbricas de transferencia de datos para favorecer y extender el alcance de los procesos de enseñanza y aprendizaje (Kuklinski, 2010). Cobos (2004) citando a Quin opina que el m-learning es el e-learning a través de dispositivos computacionales móviles, como es el caso de ordenadores portátiles de cualquier tipo y teléfonos celulares. Reconoce la capacidad de acceder a recursos de aprendizaje desde cualquier lugar, en cualquier momento, con altas capacidades de búsqueda, interacción enriquecedora, soporte poderoso para un aprendizaje efectivo y una evaluación basada en el desempeño.

S.Coop (2009) lo define como la utilización de las "tecnologías móviles" al servicio de los procesos asociados con la enseñanza y el aprendizaje. Conde (2014) lo interpreta como una evolución del e-learning que posibilita el aprovechamiento de las ventajas de las tecnologías móviles como soporte al proceso de aprendizaje (Koole, 2009) comenta que a pesar que las tecnologías móviles tienen un amplio empleo las definiciones en relación a su uso en la educación están limitadas a posiciones más técnicas que pedagógicas. En nuestro criterio esto se debe a la celeridad con la que se produce la inserción de las tecnologías y la poca investigación pedagógica en este campo. No es un fenómeno nuevo, se viene generando permanentemente a lo largo de la historia de la educación (Romero David, 2010) cita que el aprendizaje móvil se caracteriza por su capacidad de entregar contenidos de aprendizaje sin fronteras de tiempo ni espacio a través de dispositivos móviles, tales como teléfonos celulares, agendas electrónicas, pequeñas computadoras y/o todo dispositivo de mano que tenga alguna forma de conectividad inalámbrica con la finalidad de maximizar los tiempos disponibles para el aprendizaje.

Existe la tendencia a considerar que es un proceso de m-learning cuando se emplea el dispositivo móvil. No es el medio el elemento que define este tipo de aprendizaje, sino su forma de organizarlo. En este sentido empezamos a considerar que la manera adecuada de crear un verdadero proceso de m-learning es cuando partimos de la concepción de micro aprendizaje. Rizzo (2017) opina que es una forma de entregar contenido en cantidades breves, sea varias veces a la semana o diario. El micro aprendizaje debe ser considerado, en lugar de otro enfoque más entre los existentes, como una perspectiva totalmente vinculada a la educación, el aprendizaje y el m-learning. El micro aprendizaje es característico de las formas de aprender en el mundo digital, asociado a dispositivos móviles, no necesariamente un celular (Salinas, 2014). Por su parte (Romero David, 2010) considera que este se organiza en términos de momentos o episodios especiales de aprendizaje que utilizan contenidos o tareas especiales dentro de pequeñas etapas.

Hug Theo (2005) en un mapa mental recopila diferentes versiones del micro learning: entrenamiento flexible, interactivo y colaborativo a través de internet, ejercicios cortos, videos para el entrenamiento, aprendizaje situado, juegos basados en el mobile learning entre otras 


CARLOS BRAVO REYES
$>80 \quad$ UN SISTEMA DE WOOC PARA LA ACTUALIZACIÓN DOCENTE
Págs. $75-87$. DOI: $h t t p: / / d x$. doi.org/10.30972/rfce.0203255

ideas, que demuestran la variabilidad del concepto. Señala que no existe una definición precisa de esta categoría y que se pueden encontrar entre ellas diferentes dimensiones como el tiempo de duración, el contenido pequeño, que forme parte del currículo, la forma en fragmentos, el proceso con actividades separadas o integradas, la medialidad que puede ser cara a cara o mediada y por último el tipo de aprendizaje, repetitivo, reflexivo, pragmático, entre otros. La utilidad del micro aprendizaje se pone de manifiesto al facilitar la formación permanente, flexible, en cualquier horario, lugar y desde cualquier tipo de dispositivo móvil. Por ello los trabajadores desde su puesto pueden acceder a la capacitación en un tema puntual y de corta duración, al decir de (Schmidt, 2015)un aprendizaje a pedido que cruza los límites del e-learning.

El micro aprendizaje tiene su base en la concepción del e-learning que antes citamos y es una forma de organizar el aprendizaje en secuencias temporales. Se origina como respuesta a la necesidad del desarrollo personal y profesional de las personas que dedican parte de su tiempo a perfeccionar sus competencias en el campo en que se desenvuelven (Salinas, 2014) Este proceso de mejora de los conocimientos cada vez más se origina en ambientes no formales, adaptados a las necesidades y tiempo de los involucrados. Se apoya en los dispositivos móviles, su conexión a la red y el principio de la ubicuidad, en el que se puede acceder en cualquier lugar y momento a todo tipo de información. Desde el punto de vista didáctico el micro aprendizaje es una forma de organizar al aprendizaje, donde los contenidos se desarrollan en momentos breves, apoyado en métodos y medios que permitan comprender el tema tratado en poco tiempo. No es posible enmarcarlo en un tiempo exacto, pero si debe reunir la cualidad de la brevedad sin sacrificar la calidad. El micro aprendizaje se desarrolla a partir de micro contenidos y micro medios.

\section{MICRO CONTENIDOS Y MICRO MEDIOS}

La organización didáctica del micro aprendizaje abarca una variada gama de enfoques, desde no estructurado e improvisado hasta el extremo opuesto de la secuencia de acciones con una lógica interna (Hug, 2009). La mayoría de los autores consultados destacan básicamente como componentes básicos del micro aprendizaje los micro contenidos y los micro medios, pero no hacen referencias a otros de enorme significado, como los objetivos, las formas de interacción y la evaluación de los resultados. (Salinas, 2014) se refiere al micro contenido como pequeños fragmentos de información digital en permanente circulación. Señala que suele tratarse de un tema concreto, limitado en sus dimensiones, consumido rápidamente y a menudo, también limitado por el software o dispositivo empleado para su visualización.

Souza Fugisawa (2014) expresa que Nielesen en 1998 fue el primero en emplear el término de microcontenido para llamar la atención de los autores sobre la necesidad de lograr 
la claridad en los títulos, encabezados, titulares en los correos electrónicos y páginas web. Los autores consideran como ejemplos de microcontenidos los podcast, blogs, páginas de wiki, mensajes de texto o cualquier tipo de recursos audibles, visuales y verbales compartidos en la Web. Arnaud (2005) en un trabajo llamado "MicroContent is Everywhere" (el micro contenido está en todas partes) expresa que no existe una definición precisa y elabora un listado de sus rasgos. Entre estos se encuentran: pequeño, enfocado en un único tema para diferenciarlo de un título; autónomo por lo que se puede reutilizar en todo tipo de formas; direccionable donde cada pieza debe contar con una url que permita la sindicación. En estos rasgos el autor se refiere a la definición inicial de Nielsen donde incluía títulos, encabezados y titulares que se pueden encontrar en las direcciones web. La lista continúa añadiendo otros atributos como que el micro contenido es estructurado, donde la estructura puede ser mínima como un título o una url, o contener más campos, para mejorar la búsqueda. Es flexible para que el usuario pueda agregar otros campos y por último es único, refiriéndose que al ser copiado contenga toda su estructura para ser fácilmente identificado.

El micro contenido se está introduciendo cada vez más en la educación acompañada del aprendizaje móvil y del micro aprendizaje. Su característica principal es la de dividir el contenido educativo para que sea adecuado para dispositivos móviles. No se restringe a una medida de tamaño, sino a una unidad, un módulo y depende del contexto en el que se inserta (Souza Fugisawa, 2014).

Desde el punto de vista didáctico aprendemos al avanzar de una tarea a otra de manera permanente y ramificada. Considerando que esa tarea corresponde a la unidad mínima de aprendizaje, que llamaremos célula del conocimiento, entonces el micro contenido es esa célula, es la porción más pequeña que genera el aprendizaje. Los atributos que lo caracterizan son: puntual un único tema o una parte de este; preciso, evitar todo tipo de repetición, con palabras e imágenes claras, breve que se pueda asimilar en un corto período de tiempo; estructurado manteniendo la lógica del contenido y diseñado específicamente para el medio a través del cual se va a desarrollar.

Los microcontenidos se expresan a través de los micromedios. Al igual que en el caso de los micro contenidos es difícil encontrar una definición de micro medios. Pocos autores de los estudiados para este trabajo se refieren a este tipo de componente del m-learning. Salinas (2014) reconoce que cada vez vivimos en un mundo más micro media, donde los contenidos, la atención, la comunicación, los roles se están transformando en fragmentos digitales, débilmente unidos, que se reorganizan permanentemente para formar una multitud de nuevos modelos, tareas y subprocesos. Para la mayoría de los autores es más importante el micro contenido que se puede expresar en diferentes tipos de medios; desde un título, una imagen, un blog, una infografía o un micro video. En el diseño instruccional de los Wooc, el micro contenido tiene otras características y funciones diferentes. 


\section{DISEÑO INSTRUCCIONAL PARA LA CREACIÓN DE LOS WOOC}

Cuando el diseño se dirige a cursos no formales, abiertos y en línea, su organización requiere de un andamiaje diferente, que se modifica cuando se incorpora la masividad en el curso. El ideal a alcanzar es una comunidad de aprendizaje, donde el conocimiento radica en las personas, no en los datos manejados (Frida, 2015). Para la creación del sistema de Wooc trazamos la meta de crear un diseño instruccional propio Los componentes del diseño instruccional se muestran en la imagen. A continuación explicamos cada uno de ellos.

Partimos de la determinación del objetivo de cada Wooc. Debe ser puntual, preciso, considerar las características de las personas para la que es creado el Wooc, tomando en cuenta sus intereses, conocimientos previos, nivel de motivación y en especial su preparación en el manejo de la red. Otro rasgo es que debe ser alcanzado en un corto periodo de tiempo, por medio de un conjunto de acciones que se expresan en los micro contenidos.

En el sistema de Wooc los objetivos se elaboran tomando en cuenta que los profesores tienen un conocimiento general de la tecnología educativa. Emplean diferentes medios, incursionan en las aulas digitales con Moodle, emplean las redes sociales en general y en particular WhatsApp. Cada Wooc tiene un único objetivo, diseñado para ser alcanzado en un tiempo que oscila entre 60 y 90 minutos.

El segundo componente es la revisión de las características técnicas de la red, en nuestro caso WhatsApp. Esta red viene continuamente mejorando su servicio e incrementando sus posibilidades. En el primer Wooc que realizamos (Bravo Reyes, 2014) los grupos solo permitían 50 personas y hoy se triplica este número. También se modificó el formato de las imágenes entre otros elementos técnicos.

El tercer paso es el diseño y elaboración de los micro contenidos. Estos como en toda secuencia didáctica parten del objetivo y tienen como rasgos el ser breves, seguir una lógica que favorezca la rápida comprensión, evitar cualquier tipo de duda y expresados en pocas palabras. En el caso de los Wooc, el micro contenido está íntimamente ligado con los micromedios, por lo que al diseñar los primeros se debe tomar en cuenta los segundos.

El cuarto componente son los micromedios, que en el caso del diseño del Wooc son cápsulas informativas. Estas combinan texto e imágenes, construidas específicamente para cada Wooc. Se envían siguiendo un orden, se toma en cuenta el tipo de función de cada una, para determinar el tiempo en que esta es leída y analizada. Las funciones que cumplen son: organizativa, problémica, de retroalimentación y conclusiva. La imagen corresponde a una de las cápsulas del Wooc: Cómo elaborar un Wooc Por lo general cada Wooc tiene un promedio de 25 a 30 cápsulas, todas con el mismo tamaño y estilo, cada una tiene un número que corresponde al orden en que se envían.

El siguiente componente son las formas de interactuar en el Wooc, una de ellas es establecer algunas reglas para organizar la participación, otra es generar respuestas o debates 
tomando en cuenta los contenidos de cada cápsula en base a la función que cumplen. El último componente es la evaluación de los resultados y para ello partimos nuevamente del objetivo. Por lo general en el sistema de Wooc al que nos referimos, los participantes desarrollan a lo largo del mismo algún tipo de tarea, que facilita así la evaluación. Los títulos de los cursos son: Google Drive y los métodos grupales, $29+1$ utilidades didácticas de Google Drive, El crucigrama como actividad lúdica, Exámenes en línea, ¿̇Innovar en educación?, El libro impreso multimedia, Los murales interactivos, La portada de la clase, Buscar información en Google, Almacenamiento de la información, Infografías con PowerPoint, Cómo elaborar un Wooc, entre otros.

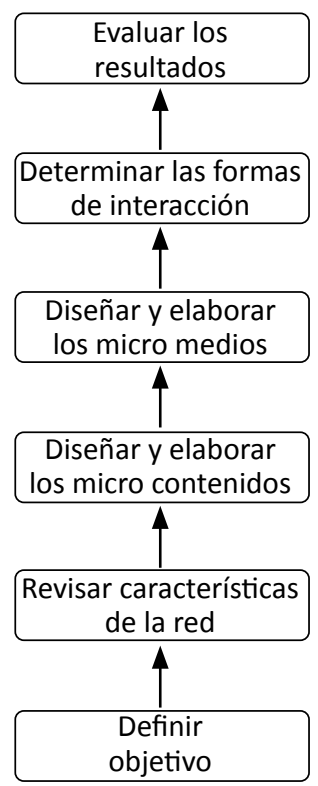

Diseño instruccional para la creación de un Wooc. Elaboración Propia.

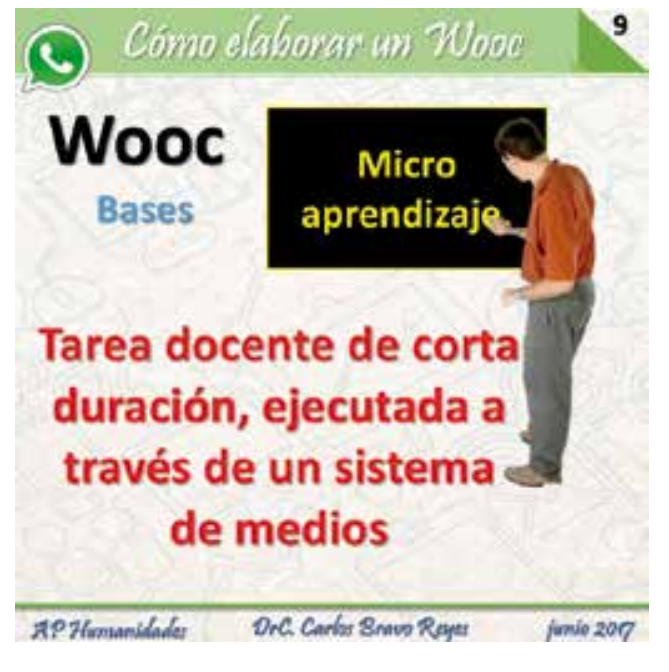

Ilustración 1Cápsula de un Wooc

\section{EVALUACIÓN DE LOS PROFESORES SOBRE EL SISTEMA DE WOOC}

Para finalizar el artículo, comentamos los resultados de un cuestionario donde los profesores participantes expresaron sus criterios sobre el sistema. Empleamos preguntas cerradas, a excepción de la última, todas utilizan una escala Likert, donde las respuestas oscilan entre nada y mucho. Las preguntas son positivas y relacionadas con la actividad de superación personal mediante los Wooc. 


\begin{tabular}{|c|c|c|c|c|c|}
\hline Pregunta & Poco (1) & 2 & 3 & 4 & Poco (5) \\
\hline $\begin{array}{l}\text { El objetivo de los Wooc se } \\
\text { puede alcanzar con facilidad }\end{array}$ & & & $3 \%$ & $18 \%$ & $79 \%$ \\
\hline $\begin{array}{l}\text { Los contenidos seleccionados para los } \\
\text { Wooc se comprenden con facilidad }\end{array}$ & & & & $24 \%$ & $76 \%$ \\
\hline $\begin{array}{l}\text { Los contenidos de los cursos le ayudan a } \\
\text { mejorar sus clases con el empleo de las Tics }\end{array}$ & & & $3 \%$ & $18 \%$ & $79 \%$ \\
\hline $\begin{array}{l}\text { Las intervenciones de los integrantes del } \\
\text { grupo de WhatsApp se corresponden con la } \\
\text { temática tratada en el Wooc }\end{array}$ & & & $9 \%$ & $36 \%$ & $55 \%$ \\
\hline $\begin{array}{l}\text { El profesor responde las preguntas } \\
\text { de los participantes }\end{array}$ & & & $3 \%$ & $6 \%$ & $91 \%$ \\
\hline $\begin{array}{l}\text { Las cápsulas informativas presentan una } \\
\text { apariencia visual agradable (relación entre } \\
\text { el texto, imágenes y color) }\end{array}$ & & & $6 \%$ & $12 \%$ & $82 \%$ \\
\hline $\begin{array}{l}\text { Las cápsulas trasmiten una información } \\
\text { adecuada (cantidad de información y } \\
\text { diseño de la cápsula) }\end{array}$ & & & $3 \%$ & $24 \%$ & $73 \%$ \\
\hline $\begin{array}{l}\text { Los Wooc en los que participó puede } \\
\text { aplicarlos en otros grupos de WhatsApp }\end{array}$ & & & $15 \%$ & $18 \%$ & $67 \%$ \\
\hline
\end{tabular}

Fuente: Elaboración propia con los datos obtenidos del procesamiento de las respuestas a la encuesta.

Como se puede apreciar todas las respuestas están por encima de la mitad porcentual. En el caso de las respuestas relacionadas con la participación en el grupo a veces estas se limitan por razones de tiempo, de no poder responder en el momento que se desarrolla el Wooc, entre otras. A las preguntas respondió la tercera parte de los integrantes del grupo.

La última de las preguntas tuvo un carácter abierto y se dirigió a recoger opiniones diversas sobre el sistema de Wooc. Entre las respuestas se señalan el que se aprendió mucho, excelente metodología, flexible, excelente instrumento para la interacción entre los docentes de la Facultad, así como también mejorar la participación de los integrantes del grupo. Es necesario señalar que este tipo de curso lo venimos realizando desde octubre del 2014 y entre varios de ellos destacan el sistema de cursos sobre la Metodología de la clase invertida” que con una convocatoria internacional reunió a más de 500 personas de 22 países (Bravo R. C., 2016).

\section{CONCLUSIONES}

Una forma de organizar la superación de los profesores en nuestra Facultad es a través de los Wooc, es decir cursos que se desarrollan por medio de WhatsApp y que se apoyan en el micro aprendizaje, donde los micro contenidos y micro medios forman parte de los componentes de su diseño instruccional. La mayoría de los profesores que participan en los Wooc 
expresan su satisfacción por esta forma de actualización en temas referidos a la tecnología y la innovación educativa tan necesarios en el trabajo docente. Los Wooc desarrollados emplean un diseño instruccional que parte del objetivo y concluye con la evaluación del mismo.

\section{REFERENCIAS BIBLIOGRÁFICAS}

Aguilar, G. V.-R. (2010). Tecnológico de Monterrey, Campus Ciudad de México. Recuperado el 07 de 2018, de http://www.iiis.org/CDs2010/CD2010CSC/CISCI_2010/PapersPdf/CA805OG.pdf

Arnaud, L. (2005). MicroContent is Everywhere. Presentation MicroLearning Conference. Innsbruck. Recuperado el o7 de 2018, de http://www.sivas.com/microcontent/articles/ microlearning2005/microlearning.pdf

Bravo Reyes, C. (octubre de 2014). Blog 366-días. Obtenido de http://366-dias.blogspot. com/2014/10/preparando-el-primer-wooc.html

Bravo, R. C. (30 de mayo de 2016). Blog 366-días. Recuperado el o7 de 2018, de Continuación del Wooc sobre la clase invertida: http://366-dias.blogspot.com/2016/o5/continuacion-delwooc-sobre-la-clase.html

Buchem, I. (2010). Microlearning: a strategy for ongoing professional development. eLearning papers. Recuperado el 07 de 2018, de www.elearningpapers.eu

Cabero, J. (2006). Bases pedagógicas del e-learning. RUSC. Universities and Knowledge Society Journal. Recuperado el o7 de 2018, de http://www.redalyc.org/pdf/780/78030102.pdf

Cobos, C. y. (2004). Vistazo general del aprendizaje móvil. VII Congreso Iberoamericano de Informática Educativa. Recuperado el 07 de 2018, de https://www.researchgate.net/ publication/251757141_Vistazo_General_del_Aprendizaje_Movil

Conde, M. Á. (2014). Recuperado el o7 de 2018, de http://www.web.upsa.es/spdeceo8/ contribuciones/128_poster_mlearningVF.pdf

Frida, B. (2015). Principios de diseño instruccional de entornos de aprendizaje apoyados con TIC: un marco de referencia sociocultural y situado. Obtenido de ILCEE: http://investigacion. ilce.edu.mx/tyce/41/art1.pdf 


\begin{tabular}{c}
\hline CARLOS BRAVO REYES \\
$>86$ \\
UN SISTEMA DE WOOC PARA LA ACTUALIZACIÓN DOCENTE \\
Págs. 75 - 87. DOI: $h t t p: / / d x$.doi.org/10.30972/rfce.0203255
\end{tabular}

Gil, F. B. (2014). Estado del Mobile Learning en España. Revista de Educación. doi: http:// dx.doi.org/10.1590/0104-4060.38646

Guerrero, A. J. (Diciembre de 2011). Observatorio Tecnológico. Recuperado el o7 de 2018, de http://recursostic.educacion.es/observatorio/web/fr/cajon-de-sastre/38-cajon-de-sastre/1026movil-learning

Hug Theo, L. M. (Ed.). (2005). Microlearning: Emerging Concepts, Practices and Technologies after e-Learning. Innsbruck University press. Recuperado el 2017, de https://www.researchgate. net/publication/246822097

Hug, T. (2009). Outline of a Microlearning Agenda. Paper. Recuperado el 10 de 2017, de www.elearningpapers.eu

Keegan, D. (2005). Mobile Learning: the next generation of learning. Distance Education International . Recuperado el o7 de 2018, de http://learning.ericsson.net/mlearning2/files/ workpackage5/book.doc

Koole, M. (2009). A Model for Framing Mobile Learnig,"Mobile Learning Transforming the Delivery of Education and Training . (A. University, Ed.) Recuperado el o7 de 2018, de http:// www.aupress.ca/books/120155/ebook/99Z_Mohamed_Ally_2009-- $\square$ MobileLearning.pdf

Kuklinski, H. P. (Noviembre de 2010). Prototipos de Mobile Open Education: Una breve selección de casos. Revista Iberoamericana de Tecnologías del aprendizaje, 125. Obtenido de http://rita.det.uvigo.es/201011/uploads/IEEE-RITA.2010.V5.N4.pdf

Pachelr, N., Bachmair, B., \& Cook, J. (2010). Mobile Learning: Structures. London: Agency, Practices.

Rizzo, C. (Octubre de 2017). Learning solutions. Recuperado el 2017, de Microlearning at John Hancock Investments Drives Results: https://www.learningsolutionsmag.com/articles/2466/ microlearning-at-john-hancock-investments-drives-results

Romero David, M.A. (Noviembre de 2010). Aprendizaje Móvil:Tendencias, Cuestiones y Retos”. Revista Iberoamericana de Tecnologías del Aprendizaje. IEEE-RITA, 5(4). Recuperado el 07 de 2018, de http://rita.det.uvigo.es/201011/uploads/IEEE-RITA.2010.V5.N4.pdf 
S.Coop, I. (2009). Mobile Learning, Análsiis prospectivo de las portenciales asociadas al Mobile Learning. Recuperado el o7 de 2018, de http://www.iseamcc.net/eISEA/Vigilancia_ tecnologica/informe_4.pdf

Salinas, J. (2014). Pasado, presente y futuro del microlearning como estrategia para el desarrollo personal. Campus virtuales, III(2). Recuperado el 2017, de http://www.uajournals. com/campusvirtuales/es/

Schmidt, A. (2015). Microlearning and the Knowledge Maturing Process: Towards Conceptual Foundations for Work-Integrated Microlearning Support. Citeseer. doi:10.1.1.70.5593

Souza Fugisawa, M. I. (2014). Educational Microcontent for Mobile Learning Virtual. Creative Education, 672-681. doi: http://dx.doi.org/10.4236/ce.2014.59079

UNESCO. (2013). Policy guidelines for mobile learning. (R. Kraut, Ed.) Paris: United Nations Educational, Scientific and Cultural Organization. Obtenido de http://unesdoc.unesco.org/ images/o021/002196/219641E.pdf

Vidal Ledo, M. J. (2015). Aprendizaje móvil. Búsqueda temática digital. Educación médica superior, 29(3). Obtenido de http://scielo.sld.cu/scielo.php?script=sci_arttext\&pid $=$ So864-21412015000300024

\section{COMO CITAR ESTE ARTÍCULO:}

BRAVO REYES, Carlos. Un Sistema de Wooc para la actualización docente. Revista de la Facultad de Ciencias Económicas - UNNE. Número 20, otoño 2018, ISSN 1668 - 6365. Págs. 75 - 87. DOI: http://dx.doi.org/10.30972/rfce.0203255

\section{CURRICULUM VITAE}

\section{Carlos Bravo Reyes}

Lic en Educación. PhD en Ciencias Pedagógicas. Profesor Titular de Tecnología Educativa en la Universidad Autónoma Gabriel René Moreno, Santa Cruz, Bolivia. Director del Observatorio de Tecnología e Innovación educativa de la UAGRM. Editor del Blog CUED de la Cátedra UNESCO de Educación a distancia de la UNED. Editor y Miembro del Consejo Científico de varias revistas científicas. cbravo@catedradigital.info 